ROBERTO MARCI, PhD

Department of Morphology, Surgery and Experimental Medicine, University of Ferrara, Ferrara, Italy

\title{
Imaging in Practice Role of imaging in endometriosis
}

\section{ABSTRACT}

Endometriosis - the presence of endometrial tissue outside the uterine cavity - is first suspected on the basis of its signs and symptoms. The diagnosis is confirmed by imaging and surgery. Imaging, particularly transvaginal ultrasonography and magnetic resonance imaging, is essential to confirm the diagnosis and guide surgical treatment.

\section{KEY POINTS}

The diagnostic evaluation should always start with transvaginal ultrasonography of the pelvic structures followed by magnetic resonance imaging, especially if deeply infiltrating endometriosis is suspected.

An inaccurate imaging evaluation may lead to an incomplete excision of lesions if the patient undergoes surgery.

Transvaginal ultrasonography and magnetic resonance imaging allow the assessment of the size, location, and extent of the lesions.

Given the multifocal nature of the disease, a thorough evaluation of all pelvic structures, including the bowel, the bladder, and the ureters, is always recommended.
32-YEAR-OLD WOMAN presents with a his$A$ tory of pelvic pain, dysmenorrhea, dyspareunia, dyschezia, and dysuria, with exacerbation of the symptoms during her menstrual cycles. Her menarche occurred at the age of 13 and her menses are regular. She has never undergone surgery and has no relevant pathologic processes. She also reports that for the past 18 months she has been unsuccessfully trying to conceive.

Two months ago, she went to the emergency department because of an acute episode of severe pelvic pain associated with abdominal cramps, vomiting, and dyschezia, occurring at the beginning of her menstrual cycle. At that time, her vital signs were within normal limits, but deep palpation of the right iliac fossa was painful. On that occasion, acute abdomen and bowel obstruction were excluded.

Now, vaginal examination reveals a bluish, painful, bulky induration in the posterior fornix. Digital rectal examination reveals a circular infiltrated area in the anterior rectal wall. Her cancer antigen 125 (CA 125) level is 230 $\mathrm{U} / \mathrm{mL}$ (normal range $0-35 \mathrm{U} / \mathrm{mL}$ ).

\section{MENSES-RELATED SYMPTOMS} AND THE DIAGNOSIS OF ENDOMETRIOSIS

The diagnosis of endometriosis should be considered in the patient described above. Many of her signs and symptoms can be associated with several diseases. However, the diagnostic hypothesis points strongly toward endometriosis, since her symptoms recur at the beginning of every menstrual cycle. ${ }^{1}$

Endometriosis is the presence of endometrial tissue outside the uterine cavity. The affected organs usually include the ovaries, fallopian tubes, ${ }^{2}$ peritoneal surface, vagina, cervix, 


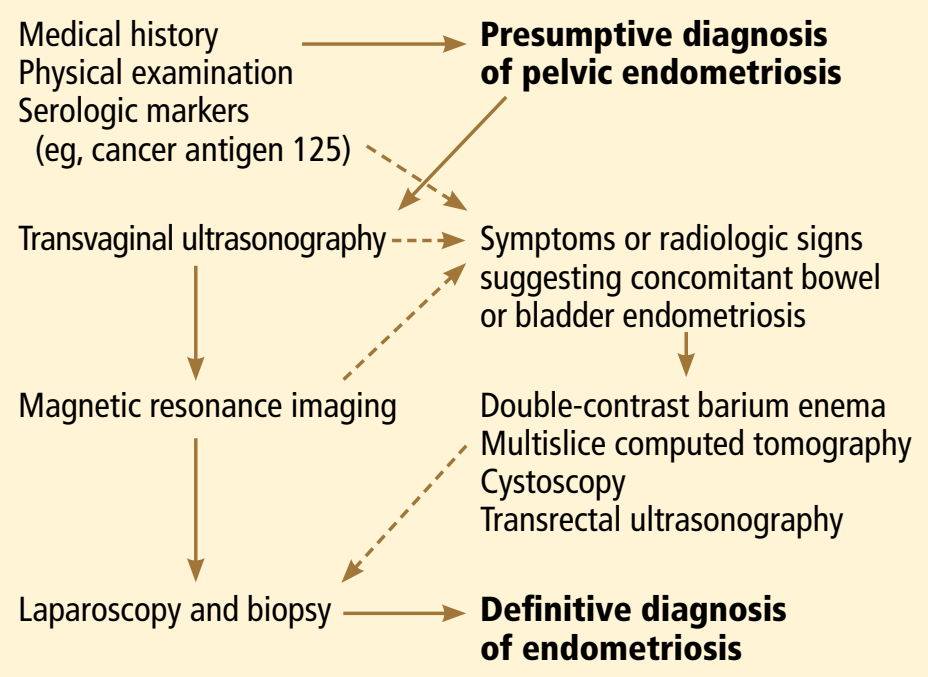

FIGURE 1. Diagnostic algorithm for endometriosis.

\section{Endometriosis} can involve any organ abdominal wall, ${ }^{3}$ scar tissue, pouch of Douglas, urinary tract, and bowel. However, any organ can be involved.

So-called deeply infiltrating endometriosis is an endometriotic lesion penetrating into the retroperitoneal space (most often affecting the uterosacral ligaments and the rectovaginal septum) or the pelvic-organ wall to a depth of at least $5 \mathrm{~mm}$ and involving structures such as the rectum, vagina, ureters, and bladder. ${ }^{4}$ Its clinical presentation is highly variable, ranging from no symptoms to severe pain and dysfunction of pelvic organs.

Endometriosis can be diagnosed with certainty only when the endometriotic lesions are observed by laparoscopy or laparotomy and after the histologic examination of surgically resected lesions (FIGURE 1). ${ }^{1}$ However, a presumptive diagnosis can be made on the basis of imaging findings, which can be useful in the differential diagnostic process (TABLE 1).

\section{EXAMINATION AND BLOOD MARKERS PROVIDE LIMITED INFORMATION}

Knowing the history of the patient, along with a physical examination that includes speculum and bimanual vaginal and rectal examination, can be helpful in the diagnostic process even if nothing abnormal is found.

Pelvic examination has a poor predictive value, as demonstrated in a study conducted by Nezhat et al ${ }^{5}$ in 91 patients with surgically confirmed endometriosis, $47 \%$ of whom had a normal bimanual examination.

CA 125 is the serologic marker most often used for diagnosing endometriosis. Levels are usually high in the sera of patients with endometriosis, especially in the advanced stages. ${ }^{6}$ However, levels increase both in the physiologic menstrual cycle and in epithelial ovarian cancers. ${ }^{7}$ Thus, the diagnostic value of $\mathrm{CA}$ 125 is limited in terms of both sensitivity and specificity.

\section{INCLUDE IMAGING IN THE DIAGNOSTIC WORKUP}

Surgical treatment is frequently offered to patients who have severe pelvic pain that does not respond to medical treatment, or in cases of infertility. Imaging investigations are mandatory both to ascertain the diagnosis and to assess involvement of internal organs before surgery. Moreover, imaging helps minimize the surgical risks.

The primary aim of the radiologic examination is to describe the precise location, the depth, and the number of pelvic endometriotic lesions. Furthermore, imaging is useful to check for endometriotic foci in pelvic organs such as the bowel, ureters, and bladder, which are often involved in the pathologic process.

Transvaginal ultrasonography and magnetic resonance imaging (MRI) can accurately delineate deeply infiltrating lesions of endometriosis that are not easily accessible laparoscopically.

\section{Transvaginal ultrasonography}

Transvaginal ultrasonography is the first-line imaging study when endometriosis is suspected: it is powerful, simple, widely available, and cost-effective. In particular, it is recommended for diagnosing endometriotic ovarian cysts (endometriomas) s, $^{8,9}$ and endometriosis of the bladder. ${ }^{10}$ However, its value for the assessment of superficial peritoneal lesions, ovarian foci, and deeply infiltrating endometriosis is questionable.

Although uncomfortable for the patient, transvaginal ultrasonography should be performed during menses, or when the pain reaches its highest level. In fact, during menstrual bleeding the endometrial implants grow and become easier to detect. 


\section{TABLE 1}

\section{Differential diagnosis of endometriotic lesions detected at imaging according to location}

\begin{tabular}{|c|c|c|}
\hline Location & Differential diagnosis & Additional information \\
\hline Bladder & $\begin{array}{l}\text { Urachal remnant } \\
\text { Epithelial tumor } \\
\text { Mesenchymal tumor }\end{array}$ & $\begin{array}{l}\text { Cystoscopic evaluation may provide } \\
\text { additional information }\end{array}$ \\
\hline $\begin{array}{l}\text { Perimetrium and round } \\
\text { ligaments }\end{array}$ & $\begin{array}{l}\text { Subserosal leiomyoma } \\
\text { Other neoplasm }\end{array}$ & $\begin{array}{l}\text { At laparoscopy, endometriosis appears as } \\
\text { red/black/white scarred lesions associated } \\
\text { or not with adhesions }\end{array}$ \\
\hline Retrocervical region & $\begin{array}{l}\text { Peritoneal metastasis } \\
\text { (ie, gastrointestinal cancer) } \\
\text { Ovarian malignancy }\end{array}$ & $\begin{array}{l}\text { Ascites and tumor mass detected elsewhere } \\
\text { in the abdominal cavity guide the diagnosis } \\
\text { to metastatic malignancies } \\
\text { At laparoscopy, retrocervical deeply infiltrating } \\
\text { endometriosis is easily recognized }\end{array}$ \\
\hline Rectovaginal space & $\begin{array}{l}\text { Bladder cancer } \\
\text { Vaginal cancer } \\
\text { Anorectal cancer }\end{array}$ & $\begin{array}{l}\text { The region is not easily accessible } \\
\text { for endoscopic viewing }\end{array}$ \\
\hline Rectosigmoid colon & $\begin{array}{l}\text { Bowel-constricting neoplasm } \\
\text { Metastatic implants to the bowel }\end{array}$ & $\begin{array}{l}\text { Colonoscopy easily detects epithelial colon } \\
\text { cancers; unlike colon cancer, deeply } \\
\text { infiltrating endometriosis starts at the serosal } \\
\text { layer and rarely affects the mucosa }\end{array}$ \\
\hline Ureters & $\begin{array}{l}\text { Ureteral cancer } \\
\text { Extrinsic ureteral obstruction due to } \\
\text { gastrointestinal or gynecologic } \\
\text { malignancies }\end{array}$ & $\begin{array}{l}\text { Correct diagnosis requires detection of the } \\
\text { epicenter of the mass } \\
\text { Renal ultrasonography is useful to reveal } \\
\text { hydronephrosis }\end{array}$ \\
\hline Ovaries & $\begin{array}{l}\text { Hemorrhagic corpus luteum } \\
\text { Dermoid cyst } \\
\text { Ovarian cancer }\end{array}$ & $\begin{array}{l}\text { Transvaginal ultrasonography, magnetic } \\
\text { resonance imaging, or computed tomography } \\
\text { usually provides sufficient information }\end{array}$ \\
\hline
\end{tabular}

Mais et $\mathrm{al}^{8}$ reported that transvaginal ultrasonography has a sensitivity of $88 \%$ in differentiating endometriomas from other ovarian masses, and a specificity of 90\% (FIGURE 2). Furthermore, its specificity is as high as that of MRI. 8,9

Endometriotic nodules detected in the uterosacral ligaments, rectovaginal septum, vagina, vesicouterine pouch, bladder (FIGURE 3), and ureters can be signs of deeply infiltrating endometriosis. Pelvic adhesions can be suspected when pelvic organs appear fixed to each other, when hyperechogenic plaques are found between the serosal surfaces of the different organs, and when the pouch of Douglas is partially or completely obliterated.
The accuracy of transvaginal ultrasonography strongly depends on the operator's skill. Furthermore, lesions of the sigmoid colon are impossible to visualize by transvaginal ultrasonography; hence, further diagnostic procedures are required. Transvaginal ultrasonography is the most accurate technique in detecting endometriotic nodules of the bladder wall in patients with urinary symptoms.

Transvaginal ultrasonography combined with color Doppler can also demonstrate the flow of urine through the ureters to the bladder, thereby ascertaining the patency of the ureters and clarifying the anatomic relationship between the ureters and any endometriotic lesions in the detrusors. ${ }^{10}$ Hydronephrosis can arise from ureteral restriction caused by 


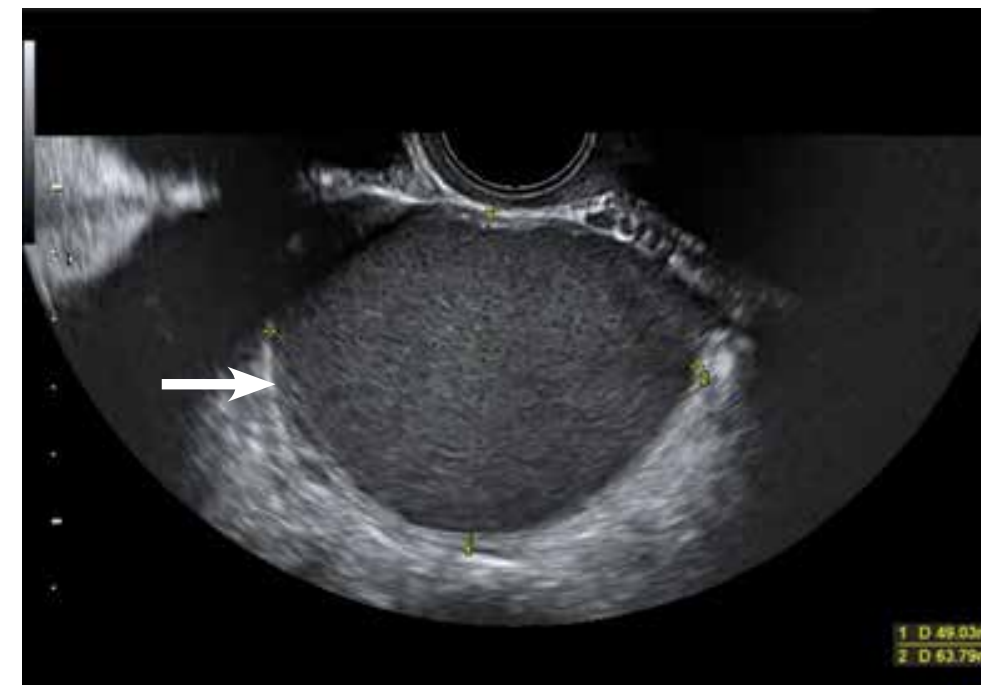

FIGURE 2. Endometriotic ovarian cysts (endometriomas) (arrow) on transvaginal ultrasonography.

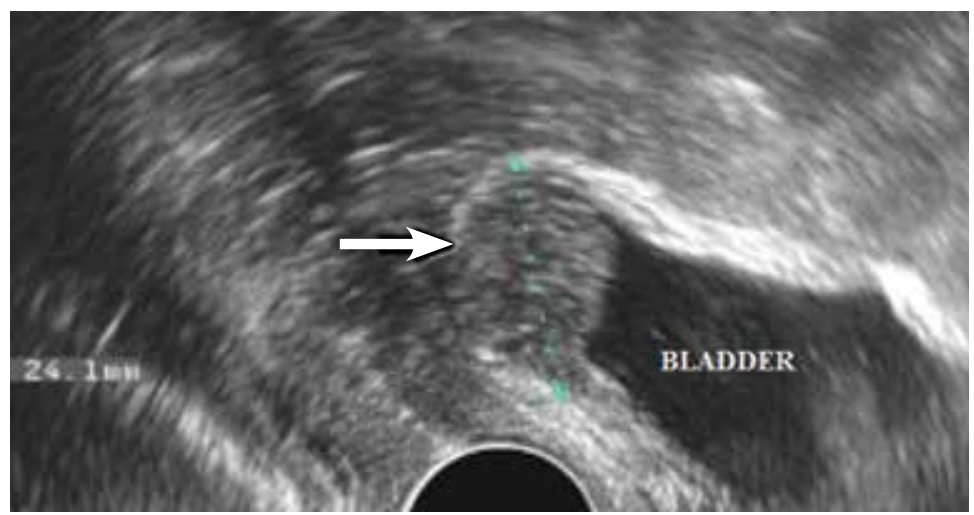

FIGURE 3. Transvaginal ultrasonography in the sagittal plane shows an endometriotic nodule in the posterior wall of the bladder (arrow).

endometriotic nodules. Thus, transabdominal ultrasonography of the kidneys is always recommended when deeply infiltrating endometriosis is suspected.

Some centers use a bowel-preparation protocol consisting of a laxative taken 24 hours before the procedure, combined with a low-residue diet and an enema 1 hour before the examination to cleanse the rectosigmoid colon of fecal content and gas, which can interfere with the visual examination of the pelvic structures. ${ }^{11}$

\section{Transabdominal ultrasonography}

Transabdominal ultrasonography can be used instead of transvaginal ultrasonography, eg, in young girls and women who have never been sexually active. When transabdominal ultra- sonography is selected, the patient should have a full bladder to maximize the visualization of the pelvic structures. However, transvaginal ultrasonography is generally more sensitive than transabdominal in detecting adnexal masses and pelvic nodules. ${ }^{12}$

\section{Magnetic resonance imaging}

MRI has been recently introduced in the diagnosis of endometriosis. MRI is less operatordependent than transvaginal ultrasonography and is more sensitive for detecting foci of deeply infiltrating endometriosis, because of its ability to completely survey the anterior and posterior compartments of the pelvis. However, its diagnostic value in cases of bladder endometriosis, superficial peritoneal lesions, and ovarian foci is still controversial. ${ }^{13-16}$

On MRI, lesions of deeply infiltrating endometriosis mainly appear as areas or nodules with regular, irregular, indistinct, or stellate margins. A distortion of the normal pelvic anatomy or the detection of a loculated fluid collection can indirectly signal the presence of adhesions.

MRI has high specificity for the diagnosis of endometriomas as a result of its ability to detect aged hemorrhagic content (FIGURE 4). ${ }^{17}$ Despite the many studies that point to the limits of MRI in detecting small endometriotic lesions, recent studies demonstrated that MRI also has good sensitivity for small peritoneal implants and adhesions. ${ }^{18,19}$ The injection of gadolinium contrast is still a debatable measure, because contrast-enhanced imaging cannot differentiate infiltrating lesions from other normal fibromuscular pelvic anatomic structures. ${ }^{15,20}$

Bowel preparation can be done with an oral laxative the day before imaging, complemented by a low-residue diet. A single dose of a ready-to-use enema is given 30 minutes before the examination to cleanse the terminal section of the intestinal tract. To avoid motion artifacts caused by bowel peristalsis, images are obtained after intramuscular injections of a myorelaxant are given, if there is no contraindication. Bowel preparation is useful to eliminate fecal residue and gas, thereby allowing proper visualization of lesions of deeply infiltrating endometriosis, but it is not routinely prescribed in all centers. ${ }^{11}$ 
In most cases, endometriotic lesions have an MRI signal intensity that comes very close to that of the surrounding fibromuscular structures. In this regard, vaginal and rectal distention and opacification using ultrasonographic gel clearly help to delineate the cervix, vaginal fornices, and vaginal wall, as well as the rectum and wall of the rectosigmoid junction (FIGURE 5). ${ }^{20}$

\section{PRESURGICAL IMAGING}

\section{Rectal endoscopic ultrasonography}

Even though it should not be included in the routine diagnostic workup, rectal endoscopic ultrasonography, using a flexible echoendoscope, is suitable in certain presurgical cases. The aim of this imaging technique is to assess the depth of bowel wall infiltration thanks to the visualization of the different layers. ${ }^{21}$

\section{Double-contrast barium enema and multislice computed tomography}

Double-contrast barium enema is extensively used for the diagnosis of bowel endometriosis, once the decision to perform surgery has been made. It allows evaluation of the degree and length of the bowel occlusion at the level of the sigmoid or high rectosigmoid tract, but it does not permit differentiation of bowel endometriosis from other pathologies.

Multislice computed tomography offers the opportunity to evaluate the depth of the lesions with excellent precision. ${ }^{22}$

The most relevant disadvantage of both procedures is the exposure of women of reproductive age to ionizing radiation. In addition, multislice computed tomography requires the administration of an intravenous iodinated contrast medium and a retrograde colonic distention with about $2 \mathrm{~L}$ of water.

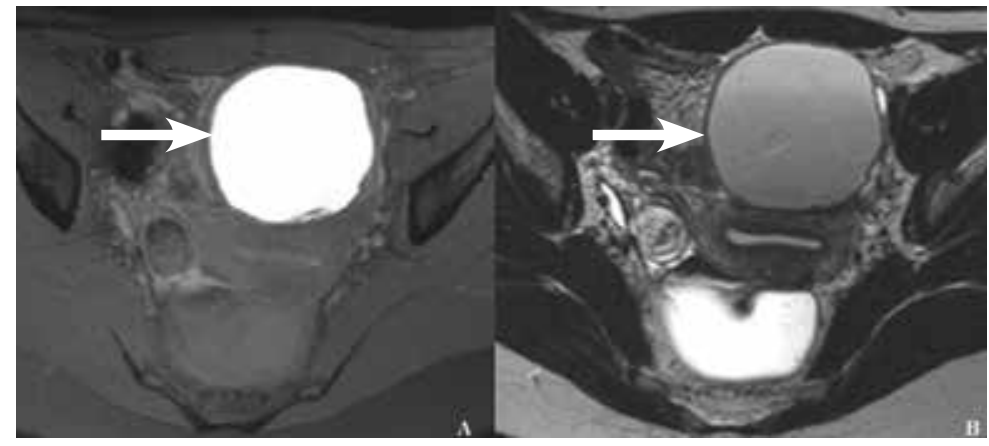

FIGURE 4. Ovarian endometrioma on magnetic resonance imaging. (A) On a fat-saturation transverse T1-weighted image, the endometrioma has high signal intensity. (B) On transverse T2-weighted image, blood-degraded product content has intermediate to low signal intensity.
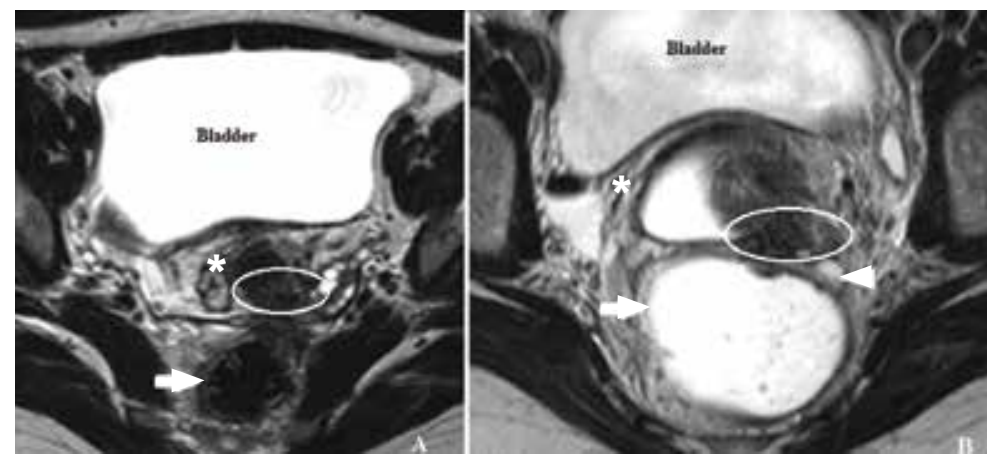

FIGURE 5. Posterior deep infiltrating endometriotic nodule on magnetic resonance imaging. (A) On a T2- weighted image with no opacification of the vagina and rectum with ultrasonographic gel, a retrocervical endometriotic nodule (white oval) has a signal intensity very close to that of the surrounding fibromuscular anatomic structures such as the rectal wall (arrow), vagina, and cervical stroma (asterisk). (B) A T2- weighted image shows the endometriotic nodule (oval) extending downward to the vaginal fornix, which appears obliterated. Only the right vaginal fornix (asterisk) is distended. Between the nodule and the anterior rectal wall, interposing fat tissue (arrowhead) is likely to represent a safety margin and a plane of dissection.

\section{REFERENCES}

1. Attaran M, Falcone T, Goldberg J. Endometriosis: still tough to diagnose and treat. Cleve Clin J Med 2002; 69:647-653.

2. Wenger JM, Soave I, Lo Monte G, Petignat P, Marci R. Tubal endometrioma within a twisted fallopian tube: a clinically complex diagnosis. J Pediatr Adolesc Gynecol 2013; 26:e1-e4.

3. Marci R, Lo Monte G, Soave I, Bianchi A, Patella A, Wenger JM. Rectus abdominis muscle endometriotic mass in a woman affected by multiple sclerosis. J Obstet Gynaecol Res 2013; 39:462-465.

4. Vercellini P, Frontino G, Pietropaolo G, Gattei U, Daguati R, Crosignani PG. Deep endometriosis: definition, pathogenesis, and clinical management. J Am Assoc Gynecol Laparosc 2004; 11:153-161.

5. Nezhat C, Santolaya J, Nezhat FR. Comparison of transvaginal sonography and bimanual pelvic examination in patients with lapa- roscopically confirmed endometriosis. J Am Assoc Gynecol Laparosc 1994; 1:127-130.

6. Barbieri RL, Niloff JM, Bast RC Jr, Scaetzl E, Kistner RW, Knapp RC. Elevated serum concentrations of CA-125 in patients with advanced endometriosis. Fertil Steril 1986; 45:630-634.

7. Bon GG, Kenemans P, Dekker JJ, et al. Fluctuations in CA 125 and CA $15-3$ serum concentrations during spontaneous ovulatory cycles. Hum Reprod 1999; 14:566-570.

8. Mais V, Guerriero S, Ajossa S, Angiolucci M, Paoletti AM, Melis GB. The efficiency of transvaginal ultrasonography in the diagnosis of endometrioma. Fertil Steril 1993; 60:776-780.

9. Guerriero $\mathbf{S}$, Mais V, Ajossa $\mathbf{S}$, et al. The role of endovaginal ultrasound in differentiating endometriomas from other ovarian cysts. Clin Exp Obstet Gynecol 1995; 22:20-22. 
10. Fedele L, Bianchi S, Raffaelli R, Portuese A. Pre-operative assessment of bladder endometriosis. Hum Reprod 1997; 12:2519-2522.

11. Chamié LP, Blasbalg R, Pereira RM, Warmbrand G, Serafini PC. Findings of pelvic endometriosis at transvaginal US, MR imaging, and laparoscopy. Radiographics 2011; 31:E77-E100.

12. Fleischer AC. Transabdominal and transvaginal sonography of ovarian masses. Clin Obstet Gynecol 1991; 34:433-442.

13. Zawin M, McCarthy S, Scoutt L, Comite F. Endometriosis: appearance and detection at MR imaging. Radiology 1989; 171:693-696.

14. Togashi K, Nishimura K, Kimura I, et al. Endometrial cysts: diagnosis with MR imaging. Radiology 1991; 180:73-78.

15. Balleyguier C, Chapron C, Dubuisson JB, et al. Comparison of magnetic resonance imaging and transvaginal ultrasonography in diagnosing bladder endometriosis. J Am Assoc Gynecol Laparosc 2002; 9:15-23.

16. Siegelman ES, Oliver ER. MR imaging of endometriosis: ten imaging pearls. Radiographics 2012; 32:1675-1691.

17. Takeuchi M, Matsuzaki K, Kubo H, Nishitani H. Magnetic resonance manifestations of endometrial cysts at $3 \mathrm{~T}$ compared with $1.5 \mathrm{~T}$. J

Comput Assist Tomogr 2008; 32:369-271.

18. Zanardi R, Del Frate C, Zuiani C, Del Frate G, Bazzocchi M. Staging of pelvic endometriosis using magnetic resonance imaging compared with the laparoscopic classification of the American Fertility Society: a prospective study. Radiol Med 2003; 105:326-338.

19. Takahashi K, Okada M, Okada S, Kitao M, Imaoka I, Sugimura K. Studies on the detection of small endometrial implants by magnetic resonance imaging using a fat saturation technique. Gynecol Obstet Invest 1996; 41:203-206.

20. Loubeyre P, Copercini M, Frossard JL, Wenger JM, Petignat P. Pictorial review: rectosigmoid endometriosis on MRI with gel opacification after rectosigmoid colon cleansing. Clin Imaging 2012; 36:295-300.

21. Bahr A, de Parades V, Gadonneix P, et al. Endorectal ultrasonography in predicting rectal wall infiltration in patients with deep pelvic endometriosis: a modern tool for an ancient disease. Dis Colon Rectum 2006; 49:869-875.

22. Biscaldi E, Ferrero S, Remorgida V, Rollandi GA. Bowel endometriosis: CT-enteroclysis. Abdom Imaging 2007; 32:441-450.

ADDRESS: Roberto Marci, PhD, Department of Morphology, Surgery and Experimental Medicine, University of Ferrara, Corso Giovecca 183, 44100, Ferrara, Italy;

e-mail: roberto.marci@unife.it

\section{Cleveland Clinic Digestive Disease Institute

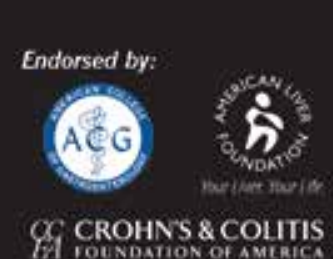

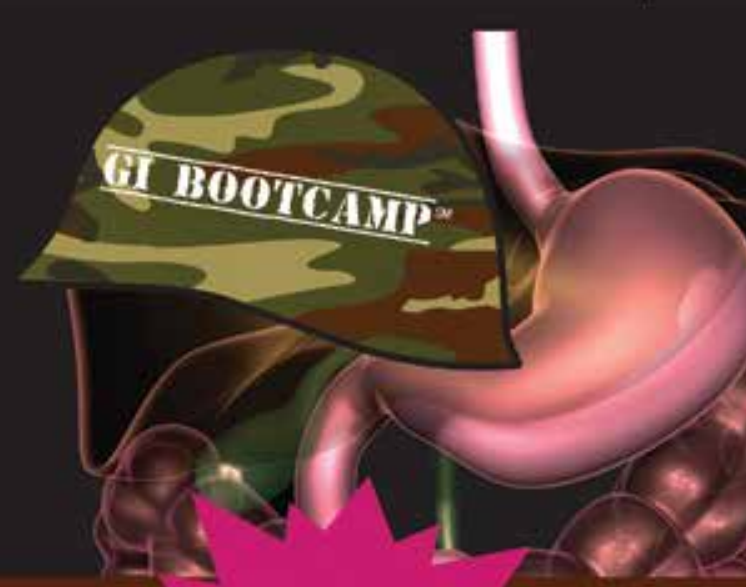

AUGUST 23 - 26, 2014

LOEWS CORONADO BAY - SAN DIEGO, CA

REGISTER TODAY! CCfCme.org/GoGIRevieW

\section{Register Now! \\ Early Bird Savings \\ Until July 15}

\section{Don't Miss This Review!}

- Get a comprehensive review for preparing for both initial certification and maintenance of recertification

AND a high-paced intensive review of the field

- Learn expert strategies for passing the $\mathrm{Gl}$ boards

- Attend sessions with high-yield material in the form of board-type questions, including gastrointestinal conditions commonly seen in practice, as well as rare but "classic" clinical vignettes

- Receive a complimentary app available for download on mobile devices with note-taking and highlighting ability

NEW FOR 2014 - Eam up to 20 points towards Maintenance of Certification by participating in two optional learning sessions 